that her disorder was only temporary, and that under treatment she would entirely recover. By means of the stomach tube the stomach was washed out thoroughly with warm water at a temperature of $100^{\circ} \mathrm{F}$. No alkali was used in the washing, as I did not wish in any. way to disturb the gastric secretions until I had examined the result from the test meal. In every case the large quantity of mucus obtained was surprising. Sips of cold water were allowed that night, and an enema of soap and water ( $\mathrm{xxx}$ ) was administered. A test meal was given next morning, and withdrawn one hour after by the stomach tube. In no case was the test meal returned, and in one patient no vestige of the test meal was obtained even by again washing out the stomach.

The filtrate from the test meal was examined, free $\mathrm{HCl}$ was present in every case, and, except in three patients, the free $\mathrm{HCl}$ and total acidity were normal; in these three exceptions the free $\mathrm{HCl}$ and total acidity were slightly below normal. The motor activity of the stomach was tested in five cases by the salol method and was found normal. Microscopic examination proved negative, and the reaction for lactic acid was never present.

I therefore came to the conclusion that the drugs bismuth carbonate, cerium oxalate, etc., recommended in textbooks were not required for these 16 cases of hyperemesis gravidarum. For several days the patients were put on peptonized milk and milk and soda, and were thereafter given light diet. No hard and fast rule was laid down as to the day of treatment on which the patient was advanced to a light or a full diet; each case was considered separately and dieted accordingly. The patients were not allowed up until they had been on light diet for two days. A powder containing 1 grain of mercury-with-chalk powder and 3 of sodium bicarbonate was given thrice daily. For chronic constipation, in conjunction with the above powder I found magnesium sulphate in hot water every second morning the best aperient. If a satisfactory motion was not obtained, then a soap and water enema ( $3 \times x x)$ was given. The septic condition of the mouth was treated with a mouth wash of carbolic acid solution 1 in 80 , used frequently and always immediately before and immediately after food. The patients were encouraged to drink copiously of milk and soda, weak tea, and imperial drink.

This treatment was quite successful in the 16 cases, the average duration of residence in the hospital being seventeen days. One patient was treated in hospital for about ten days with the textbook drugs without improvement. She was then treated as above and at once improved. She was dismissed well within three weeks thereafter. She had no return of vomiting, went to full term, and was delivered of a healthy child. In another case there was retroversion of the uterus with tendency to prolapse; the uterus was not interfered with, and she made a complete recovery. Five cases presented a woll marked acetone odour. Several cases returned to hospital at full time in labour, and had had no return of the vomiting.

A point worthy of notice is that 15 of these cases were admitted to hospital during the months of November and December, 1909, and January and February, 1910. The weather in Glasgow and neighbourhood during the first three months mentioned was extremely inclement, and did not conduce to out-of-door exercise. The continuance of fog and excessive cold was such that the death-rate in the city was on several occasions over 30 per 1,000 a week. With improved weather 2 cases only were sent into hospital from March 1st to May 31st, 1910. I am of opinion that in hyperemesis gravidarum, as in eclampsia, prophylactic treatment is of prime importance, and recommend the following as the important points :

1. Bowels to be moved at least once per diem.

2. Prevention of oral sepsis.

3. Good plain well-cooked food, daintily served at regular hours.

4. Kidneys and skin to be kept active.

5. Gentle exercise in the open air daily if weather at all suitable, and a good supply of fresh air night and day.

Summary of treatment when hyperemesis gravidarum has developed:

1. Wash out the stomach.

2. Bowels, kidneys, and skin to be kept active.

3. Nutriment at regular hours.

4. Fresh air night and day.

\section{THE TREATMENT OF AORTIC ANEURYSMS.}

\section{BY}

ALBERT ABRAMS, A.M., M.D., SAN FRANCISCO, CALIFORNIA, U.s.A.

Aortic Reflexes.

IN $1904^{1}$ I demonstrated that, if one made forcible concussion of the seventh cervical spine, the normal aorta could be brought to contraction (aortic reflex of contraction), and that, if the last four dorsal vertebral spines were struck a series of sharp vigorous blows, a dilatation of the aorta (aortic reflex of dilatation) ensued. A like response was observed in aortic aneurysms.

It occurred to me that the aortic reflex of contraction could be utilized in the treatment of aortic aneurysms. Forty cases of aneurysm of the thoracic and abdominal aorta have thus far been treated, and have all been symptomatically cured in a brief period of time. Some of the patients were seen after a period of four years with absolutely no recurrence of symptoms.

I desire to emphasize the following facts :

1. Aneurysm was only diagnosed when the classic symptoms of the disease could be demonstrated by physical and skiascopic methods of examination.

2. Pressure symptoms were present and the disease was in an advanced stage in all the patients.

3. The only treatment employed was concussion of the seventh cervical spine. Not even rest was enjoined, although the patients were instructed to avoid straining at stool and exertion.

In the therapeutic elicitation of the aortic reflex of contraction an apparatus which gives a percussion stroke must be employed ; all other motions, such as oscillations, shaking, and friction, interfere with results. The pad of the apparatus can be applied directly to the seventh cervical spine, or indirectly if the skin is sensitive, by interposing a piece of lint or linoleum. The daily sittings of concussion last about fifteen minutes, but the treatment must be interrupted to avojd irritation of the skin. If a suitable apparatus is not available, a pleximeter of rubber, wood, ivory, or linoleum is applied to the seventh cervical spine and struck a series of rapid and moderate blows by means of a strong percussion hammer. I use the plexor of Déjerine."

'The subsidiary centre of the vaso-constrictor nerves of the aorta is in the spinal cord, in proximity to the spinous process of the seventh cervical vertebra (third dorsal segment of the spinal cord); and by stimulation of this centre by concussion the normal as well as the abuormal aorta may be brought to contraction. Other stimuli have been tried, but the most effective stimulant to elicit the reflex is concussion. The aortic reflexes are mediated through the vagus; they are accentuated after a hypodermic injection of pilocarpin (0.0065 gram) and inlibited by atropin ( 0.001 gram).

To illustrate the rapidity of results achieved by this method, three cases will be cited :

Patient of 1)r. L. St. John Hely.2-Aneurysmal tumour which protruded from the chest measured $2 \frac{1}{2} \mathrm{in}$. in diameter at the base. After the fourth treatment there was an absolute disappearance of all symptoms (incessant dyspnoea, cyanosis, choking, cough). At the end of a week the size of the tumour was reduced 25 per cent., and further treatment was discourfinned, insomuch as the patient regarded himself as cured. This patient was observed after fourteen months with no retura of subjective symptoms.

Patient of Dr. Hubert $N$. Rovell.-Pronounced pressure sym-

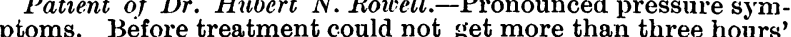
ptoms. Before treatment could not get more than three hours' sleep at night, owing to paroxysmal attacks of coughing and choking. After the first treatment he could sleep the entire night, and after two weeks' treatment the patient was prac-
tically well, and had gained $10 \mathrm{lb}$. in weight. 'This patient tically well, and had gained $10 \mathrm{lb}$. in weight. This patient
was seen after three years, with absolutely no return of symptoms.

Patient of Dr. Wm. Clark.-In addition to the conventional objective signs of aneurysm, there was a pronounced exophthalmos. The subjective signs were dyspnoea, incessant cough, dysphonia, and the patient was subjectively conscious of the aortic bruit. All the subjective symptoms disappeared within several days after treatment was commenced, and after five weeks' treatment the patient was practically discharger After a lapse of more than two years the patient is absolutely well.

- The method of treatment is discussed in detail in the author's book, Spondylotherapy, 1910, Philopolis Press, San Francisco. 
Conclusions.

1. Elicitation of the aortic reflexes aid the diagnosis of aortic aneurysm.

2. Elicitation of the aortic reflex of contraction results in the symptomatic cure of aortic aneurysms, and the results are so astonishingly rapid that one is warranted in concluding that the method is practically a specific.

3 . If results are not achieved, there is either an error in diagnosis or the aneurysm is beyond restitution.

4. By aid of skiascopy, skiagraphy, percussion, and auscultation one may determine objectively the improvement of the patient, although the reduction in the area of the aneurysmal tumour is not in proportion to the amelioration of the subjective symptoms.

5. It is reasonable to assume that like results may be achieved in aneurysms. of other vessels, insomuch as plethysmography, sphygmography, and other observations by the author show that all the arteries of the body are brought to contraction by definite stimuli applied to the seventh cervical spine.

REFERENCEA

1 Abrams, American Medicine, April 2nd, 1904; Medical Record, September 16th, 1905. 2 Abrams's Method in the Treatment of Aneurysm of the Aorta, Medical Record, May 21st, 1910.

\section{HYPERTROPHIED PAPILLAE OF MORGAGNI CAUSING RÉCTAL SYMPTOMS.}

\author{
BY
}

\section{A. CAMPBELL MAGAREY, M.B.Adelaide,} M.R.C.S.ENG.,

HOUSE-SURGEON TO ST. MARK'S HOSPITAL FOR CANCER, FISTULA, AND OTHER DISEASTS OF THE RECTOM.

THE papillae of Morgagni are situated at the ano-rectal junction, and though absent in the majority, are yet present in a considerable proportion of individuals. They are normally small white glistening papillae, pyramidal or triangular, with the apices directed upwards and inwards towards the lumen of the gut. In number they vary from one to six, and are situated either at the upper extremities of the columns of Morgagni or on the edges of the valves intervening between the upper ends of these columns. Their length varies from $\frac{1}{8}$ in. to $\frac{1}{3}$ in.

Stroud ${ }^{1}$ describes them as consisting of myelinated and non-myelinated nerve fibres, with a considerable number of ganglion cells and very little connective tissue. They are covered by a stratified epithelium.

When hypertrophied, the papillae much resemble in shape and size the writing end of an ordinary lead pencil. They must be distinguished from the small fibrous polyp which sometimes forms on the top of an internal haemorrhoid.

Stroud has shown that these papillae are peculiar to man, occurring at all periods of life, having been seen in the fetus, and in patients of advanced years. -They are not found in the lower animals, though search has been made for them. They are considered to be accessory sense organs, aiding and warning the anal sphincters. When these functions become unduly developed and the papillae are hyperaesthetic, they give rise to symptoms. Andrews ${ }^{2}$ speaks of them as "special sense organs of the rectum," and mentions their removal as one of the causes of incontinence which sometimes occurs subsequent to Whitehead's operation for haemorrhoids.

While realizing that the out-patient department of a rectal hospital is not the place to investigate the normal rectum, I have examined fifty patients with the speculum, as far as possible in consecutive order. In this series papillae were present in nineteen instances, or 38 per cent. of the cases. The age of the patients examined ranged from 12 to 68 years with an average of 39 years, and the average age of those in whom papillae were present was 38 years. The number of papillae varied from one to six with an average of two.

The symptoms attributable to the hypertrophied papillae are as follows :

1. Irritation.

This is by far the most frequent cause of complaint, and in a large percentage of patients suffering from pruritus ani these hypertrophied papillae greatly augment the trouble, if indeed they are not the sole cause. This is demonstrated by the beneficial effect of cauterization or removal, as in practieally all cases the patients are greatly re'ieved and many cured. In a series of 53 patients suffering from pruritus ani attending the out-patient department of St. Mark's during the six months June to November, 1910, hypertrophied papillae were present in 13 cases-that is, in 25.6 per cent.

The papillae were cauterized more or less thoroughly in all these cases, and in only one was there lack of improvement. The majority of cases made two subsequent attendances, reporting great relief, and then ceased to attend. One case of a Jewess was particularly marked. She had irritation of eight months' duration; she had the typical hard plicated skin surrounding the anus common in people who suffer from irritation. Two papillae were cauterized, and from that time the irritation ceased, and a month later she had suffered no return, and discharged herself cured. It has yet to be proved whether this benefit is to be permanent.

\section{Pain.}

Durfng the same six months $I$ have collected 12 cases in which the complaint was of $(a)$ pain in the lower part of the back, aching in character; $(b)$ shooting pain in the rectum; $(\dot{c})$ intense pain when the bowels acted, differing from that produced by fissure or fistula in that, instead of a sharp burning or stabbing pain of considerable duration, it is a sensation of sharp pricking which soon passes' off. Careful examination in all these cases revealed nothing except hypertrophied papillae.

In every case cauterization afforded relief. Of the three types of pain mentioned, the shooting pain in the rectum, extending up to the base of the spine, is the most usual, while the dull aching pain and that present when the bowels act seem to be about equal in frequency.

\section{Sensation of Mass in the Rectum.}

This is a very common symptom, and, as a rule, the patient says that he feels as though the bowel protrudes on defaecation, but that nothing actually: emerges. In these cases, again, the symptoms disappear on cauterization of the papillae. In all these 12 cases the patients complained of pain of one of the three types above mentioned, and also of the sensation of a lump in the rectum.

When much hypertrophied the papillae can be readily recognized by digital examination as small, shotty nodules. They can always be seen at the ano-rectal junction by means of a speculum.

\section{Treatment.}

Cauterization is undoubtedly the simplest, quickest, and most effective method of destroying the papillae; other methods I have tried, such as snipping off with scissors and destruction with nitric acid and other caustics, have no advantages, and are not so complete. The pain attendant upon cauterization is slight, and may be abolished by insufflating some analgesic powder through a speculum and allowing it to remain in the rectum for ten or fifteen minutes, after which cauterization is absolutely painless. The method used at St. Mark's Hospital is to pass a Kelly's speculum into the rectum and then withdraw it until the papillae stand up at the inner end, when they can be successively touched with the electric thermocautery. One application is generally sufficient, but in three cases I have recauterized two weeks later.

It is remarkable that none of the works on rectal surgery gives any attention to the symptoms caused by these papillae. They were mentioned as a separate disease in the annual report of St. Mark's Hospital for the first time in 1908, when eleven cases were treated as in-patients.

In one book of rectal surgery ${ }^{3}$ only are they men. tioned, near the end of a long list of causes of pruritus, but I have been unable to find any mention of them in connexion with other symptoms.

I am much indebted to Messrs. Mummery, Baldwin, and Gordon Watson, the surgeons in charge of the out-patients, for their kindness in allowing me to use their cases, and particularly to Mr. Gordon Watson for his suggestion that I should collect these cases, and for the assistance he has given in these investigations. 